\title{
Gauge fields, point interactions and few-body problems in one dimension
}

\author{
S.Albeverio ${ }^{1,2}$, S-M.Fei ${ }^{1,3}$, and P.Kurasov ${ }^{1,4}$ \\ ${ }^{1}$ Institut für Angewandte Mathematik, Universität Bonn, D-53115 Bonn \\ ${ }^{2}$ SFB 611; BiBoS; CERFIM (Locarno); Acc.Arch., USI (Mendrisio) \\ ${ }^{3}$ Dept. of Mathematics, Capital Normal University, 100037 Beijing \\ ${ }^{4}$ Dept. of Mathematics, Lund Institute of Technology, 22100 Lund \\ E-mail: albeverio@uni-bonn.de, fei@uni-bonn.de, kurasov@maths.lth.se
}

\begin{abstract}
Point interactions for the second derivative operator in one dimension are studied. Every operator from this family is described by the boundary conditions which include a $2 \times 2$ real matrix with the unit determinant and a phase. The role of the phase parameter leading to unitary equivalent operators is discussed in the present paper. In particular it is shown that the phase parameter is not redundant (contrary to previous studies) if non stationary problems are concerned. It is proven that the phase parameter can be interpreted as the amplitude of a singular gauge field. Considering the few-body problem we extend the range of parameters for which the exact solution can be found using the Bethe Ansatz.
\end{abstract}

Keywords: point interactions, Schrödinger operator, boundary conditions, few-body system.

1 Introduction.

The problem of describing point interactions for one dimensional Schrödinger operators attracts considerable attention by researchers in various disciplines, since the spectral structure of such operators can easily be investigated and the eigenfunctions can be calculated explicitly. This problem is similar to the one of describing point interactions for the Laplace operator in $\mathbf{R}^{3}$ analyzed first by F.A.Berezin and L.D.Faddeev [7. From the mathematical perspective the problem in one dimension is not complicated, since all operators with point interactions are extensions of one symmetric operator with deficiency indices $(2,2)$ and their resolvents can be calculated using Krein's formula 3, 6, 4, 12, 14, 20. The problem of describing all these operators as singular perturbations of the free second derivative operator has been solved in [14]. It has been proven that the four parameter family of point interactions can be realized as a combination of a singular potential, a singular density and a singular gauge field. Therefore all point interactions for the second derivative operator in one dimension have been classified. In particular the one parameter family of point interactions corresponding to the heuristic Schrödinger operator with a $\delta^{\prime}$-potential has been found, supporting the conclusions of [13. The distribution theory with discontinuous test functions has been used to justify the proofs [14 16. This approach has been generalized to include finite rank perturbations of arbitrary self-adjoint 
operators [5]. The relations between this description of point interactions and the symmetry properties of the corresponding heuristic Hamiltonians have been clarified in 2.

F.A.B. Coutinho et all [10 pointed out that changing one of the parameters describing these point interactions (the so-called phase parameter) one gets a family of operators which are unitary equivalent. In accordance to the classification given in [14] this parameter corresponds to a singular gauge field concentrated at the origin. Therefore it is not surprising that this parameter leads to the one-dimensional family of unitary equivalent operators, since the gauge field in one dimension can easily be removed by a unitary transformation. In the current paper we further clarify these relations and show that the phase parameter is not redundant as far as the nonstationary problem is concerned. These results are generalized for the case of a system of $N$ one dimensional particles interacting via two-body point interactions discussed recently in [1, 11, 15. following the main ideas of [19]. It is proven that the scattering states and bound state functions can be calculated for a $2\left(\begin{array}{l}N \\ 2\end{array}\right)+1$ parameter family of Hamiltonians, which includes the system of one dimensional particles with delta two-body interactions as a special case. It is well known that the ground state eigenfunction for $N$-particle Hamiltonians with $\delta$-interactions possesses a boson like symmetry [17, 18, 21].

2 Unitary equivalence of operators with point interactions

The family of point interactions for the one dimensional Schrödinger operator $-\frac{d^{2}}{d x^{2}}$ can be described by unitary $2 \times 2$ matrices via von Neumann formulas for self-adjoint extensions of symmetric operators, since the second derivative operator restricted to the domain $C_{0}^{\infty}(\mathbf{R} \backslash\{0\})$ has deficiency indices $(2,2)$ (see 3, 6, 9, 20, ) (We supposed here for simplicity that the point interaction is situated at the origin). It is more convenient to describe the same family of operators by certain boundary conditions at the origin imposed on the functions from the domain of any self-adjoint extension. In what follows we are going to consider only the self-adjoint extensions that cannot be presented as an orthogonal sum of two self-adjoint operators acting in $L_{2}(-\infty, 0)$ and $L_{2}(0, \infty)$ (the half axes cannot be separated). Then the boundary conditions describing the operator have the following form

$$
\left(\begin{array}{c}
\psi(+0) \\
\psi^{\prime}(+0)
\end{array}\right)=e^{i \varphi}\left(\begin{array}{ll}
a & b \\
c & d
\end{array}\right)\left(\begin{array}{c}
\psi(-0) \\
\psi^{\prime}(-0)
\end{array}\right)
$$

where $\varphi, a, b, c, d \in \mathbf{R}, a d-b c=1$. Let us denote by $L_{\varphi, a, b, c, d}$ the corresponding self-adjoint operator defined on the functions from $W_{2}^{2}(\mathbf{R} \backslash\{0\})$ satisfying the boundary conditions (11). It has been noted in [10] that the operators $L_{\varphi, a, b, c, d}$ and $L_{0, a, b, c, d}$ are unitary equivalent. In fact, consider the function

$$
\chi(x)=\left\{\begin{array}{cc}
1, & x<0 \\
e^{i \varphi}, & x \geq 0 .
\end{array}\right.
$$

Multiplication by the function $\chi(x)$ (which has modulus 1) establishes the unitary equivalence between the operators $L_{\varphi, a, b, c, d}$ and $L_{0, a, b, c, d}$ :

$$
\chi^{*} L_{\varphi, a, b, c, d} \chi=L_{0, a, b, c, d} .
$$


The function $\chi$ is not continuous. Therefore the domains of the operators $L_{\varphi, a, b, c, d}$ and $L_{0, a, b, c, d}$ do not coincide. The unitary equivalence of the operators implies that all important physical quantities determined by these operators are essentially the same as long as the stationary problem is concerned.

The authors of [10] concluded that the parameter $\varphi$ is redundant, since it produces no interesting effect. In what follows we are going to show, however, that this parameter can play an important role in the study of nonstationary problems and for these problems interesting effects can be observed. Consider for example the following equation

$$
L_{\varphi(t), 1,0,0,1} \psi(x, t)=\frac{1}{i} \frac{\partial}{\partial t} \psi(x, t)
$$

where the phase parameter $\varphi$ depends on the time (time-dependent gauge field). The unitary transformation (3)

$$
\psi(x, t) \mapsto \phi(x, t)=\chi^{*} \psi(x, t),
$$

which is time dependent as well, maps the equation (4) into the Schrödinger equation with time dependent step potential

$$
\left(L_{0,1,0,0,1}-\Theta(x) \varphi^{\prime}(t)\right) \phi(x, t)=\frac{1}{i} \frac{\partial}{\partial t} \phi(x, t),
$$

where $\Theta=\left\{\begin{array}{ll}1, & x \geq 0 \\ 0, & x<0\end{array}\right.$. The operator $L_{0,1,0,0,1} \equiv L$ coincides with the free second derivative operator on the line. Consider now the simplest case where the derivative of $\varphi$ is equal to a negative constant $\varphi^{\prime}(t)=-h<0$. Then the latter equation coincides with the Schrödinger equation with the time independent step potential $h \Theta(x)$. For this problem one can observe total reflection of incoming plane waves with small energies $0<k^{2}<h$. This is in contrast to the free evolution determined by

$$
L \phi(x, t)=\frac{1}{i} \frac{\partial}{\partial t} \phi(x, t) .
$$

This example illustrates the importance of the phase parameter $\varphi$ describing the family of point interactions for the second derivative operator for nonstationary problems.

3 Gauge field and point interactions

We are going to discuss the physical interpretation of the phase parameter $\varphi$. It has been proven in [14] that this parameter is related to a singular gauge field concentrated at the origin. The following heuristic operator has been considered in [14,

$$
L_{\alpha}=\left(i \frac{\partial}{\partial x}+\alpha \delta(x)\right)^{2}-\alpha^{2} \delta(x)^{2} \equiv-\frac{\partial^{2}}{\partial x^{2}}+i \alpha\left(2 \frac{\partial}{\partial x} \delta(x)-\delta^{(1)}(x)\right),
$$

where by $\delta^{(1)}$ we denote the first (generalized) derivative of the $\delta$-function. It has been proven in 14] that the latter operator coincides with the second derivative operator $L_{\alpha}=-\frac{\partial^{2}}{\partial x^{2}}$ defined on the domain

$\operatorname{Dom}\left(L_{\alpha}\right)=\left\{\psi \in W_{2}^{2}\left(\mathbf{R} \backslash\{0\}:\left(\begin{array}{c}\psi(+0) \\ \psi^{\prime}(+0)\end{array}\right)=\frac{2+i \alpha}{2-i \alpha}\left(\begin{array}{cc}1 & 0 \\ 0 & 1\end{array}\right)\left(\begin{array}{c}\psi(-0) \\ \psi^{\prime}(-0)\end{array}\right)\right\}\right.$. 
We get boundary conditions from the family (1) if we put

$$
e^{i \varphi}=\frac{2+i \alpha}{2-i \alpha}
$$

and $a=d=1, b=c=0$.

The whole four parameter family of boundary conditions for the second derivative operator has been classified in [14 in terms of operators with singular interactions. The singular interactions included a singular potential, a singular density and a singular gauge field. It has been also proven in [14 that a nontrivial phase in the boundary conditions appears if and only if the singular gauge field is present.

The fact that the operators with and without singular magnetic field are unitary equivalent is not surprising and reminds us about the well known fact that the one dimensional Schrödinger operator with a smooth gauge field is unitarily equivalent to the second derivative operator ${ }^{1}$. In fact let us consider the one dimensional magnetic Schrödinger equation

$$
\left(i \frac{\partial}{\partial x}+a(x)\right)^{2} \psi(x, t)=\frac{1}{i} \frac{\partial}{\partial t} \psi(x, t)
$$

where $a \in C^{1}(\mathbf{R}) \cap L_{1}(\mathbf{R})$. Then the domain of the operator

$$
L(a)=\left(i \frac{\partial}{\partial x}+a(x)\right)^{2}
$$

coincides with the Sobolev space $W_{2}^{2}(\mathbf{R})$, and the operator $L(a)$ is self-adjoint on this domain. Consider the following new unitary function

$$
\chi_{a}(x)=e^{i \int_{-\infty}^{x} a\left(x^{\prime}\right) d x^{\prime}} .
$$

The operator of multiplication by $\chi$ establishes the unitary equivalence of the operator $L(a)$ and the free second derivative operator $L$

$$
\chi_{a}^{*} L(a) \chi_{a}=L .
$$

One can observe that for small $\alpha$ and $\varphi,|\alpha|,|\varphi| \ll 1$, formula (8) reduces to

$$
\varphi=\alpha+O\left(\alpha^{2}\right) .
$$

This is not surprising, since the operators $L_{\alpha}$ and $L(\alpha \delta)$ differ heuristically by a term proportional to $\alpha^{2}$. Then substituting $a(x)=\alpha \delta(x)$ heuristically into the formula for $\chi_{a}(x)$ we get

$$
\chi_{\alpha \delta(x)}=e^{i \alpha \Theta(x)}=\left\{\begin{array}{cc}
1, & x<0 \\
e^{i \varphi}, & x \geq 0
\end{array}\right.
$$

which just coincides with (2).

4 Few-body problem

\footnotetext{
${ }^{1}$ Physicists like to express this fact by saying that "Gauge field does not exist in one space dimension"
} 
The unitary transformation discussed in the previous section for two-body problems with point interactions can easily be generalized to include the case of few body interactions. Consider a system of $N$ equal one dimensional quantum particles with the coordinates $x_{i} \in \mathbf{R}, i=1,2, \ldots, N$ interacting via two body $\delta$-function potentials. This system is described by the operator

$$
\mathcal{L}_{N}=-\sum_{i=1}^{N} \frac{\partial^{2}}{\partial x_{i}^{2}}+\frac{c}{\sqrt{2}} \sum_{i<j} \delta\left(x_{i}-x_{j}\right)
$$

(with $c$ a real parameter) which is exactly solvable in the sense that all its eigenfunctions can be calculated explicitly as combinations of plane waves or exponential functions [19, 21]. The scattering states for this operator can be calculated using an important analogy with the diffraction problem on a system of wedges [8, 15]. The book [6] contains the most complete reference list on this subject.

Let us denote by $x_{i j}$ the relative Jacobi coordinate associated with the cluster formed by the particles $i$ and $j: x_{i j}=\sqrt{2}\left(x_{i}-x_{j}\right)$ and by $y_{i j} \in$ $\mathbf{R}^{N-1}$ the relative coordinates of all other particles with respect to the cluster's center of mass. It is easy to show that the operator $\mathcal{L}_{N}$ coincides with the Laplace operator $\mathcal{L}_{c}=-\sum_{i=1}^{N} \frac{\partial^{2}}{\partial x_{i}^{2}}$ defined on the domain of functions from $\psi \in W_{2}^{2}\left(\mathbf{R}^{N} \backslash \cup_{i<j}\left\{x_{i}=x_{j}\right\}\right)$ satisfying the following boundary condition on each hyperplane $l_{i j}=\left\{\left(x_{i j}, y_{i j}\right) \in \mathbf{R}^{N}: x_{i j}=0\right\}$

$$
\left.\left(\begin{array}{c}
\psi\left(x_{i j}, y_{i j}\right) \\
\frac{\partial \psi}{\partial x_{i j}}
\end{array}\right)\right|_{x_{i j}=+0}=\left.\left(\begin{array}{cc}
1 & 0 \\
c & 1
\end{array}\right)\left(\begin{array}{c}
\psi\left(x_{i j}, y_{i j}\right) \\
\frac{\partial \psi}{\partial x_{i j}}
\end{array}\right)\right|_{x_{i j}=-0} .
$$

Consider now the Laplace operator $\mathcal{L}_{c, a_{i j}, \varphi_{i j}}=-\sum_{i=1}^{N} \frac{\partial^{2}}{\partial x_{i}^{2}}$ defined on the domain of functions from $\psi \in W_{2}^{2}\left(\mathbf{R}^{N} \backslash \cup_{i<j}\left\{x_{i}=x_{j}\right\}\right)$ satisfying the following boundary condition on each hyperplane $l_{i j}=\left\{\left(x_{i j}, y_{i j}\right) \in \mathbf{R}^{N}: x_{i j}=0\right\}$

$$
\left.\left(\begin{array}{c}
\psi\left(x_{i j}, y_{i j}\right) \\
\frac{\partial \psi}{\partial x_{i j}}
\end{array}\right)\right|_{x_{i j}=+0}=\left.a_{i j} e^{i \varphi_{i j}}\left(\begin{array}{cc}
1 & 0 \\
c & 1
\end{array}\right)\left(\begin{array}{c}
\psi\left(x_{i j}, y_{i j}\right) \\
\frac{\partial \psi}{\partial x_{i j}}
\end{array}\right)\right|_{x_{i j}=-0}
$$

where $\varphi_{i j} \in[0,2 \pi)$ and $a_{i j}>0$ are real parameters ${ }^{2}$. Altogether there are $2\left(\begin{array}{c}N \\ 2\end{array}\right)+1$ real parameters that determine the operator $\mathcal{L}_{c, a_{i j}, \varphi_{i j}}$. The operator $\mathcal{L}_{c, a_{i j}, \varphi_{i j}}$ is self-adjoint in $L_{2}\left(\mathbf{R}^{N}\right)$ only if all $a_{i j}=1$. It is easy to show that the operators $\mathcal{L}_{c}$ and $\mathcal{L}_{c, a_{i j}, \varphi_{i j}}$ are similar. Consider the functions

$$
\chi_{i j}\left(x_{i j}, y_{i j}\right)= \begin{cases}1, & x_{i j}<0 \\ a_{i j} e^{i \varphi_{i j}}, & x_{i j} \geq 0\end{cases}
$$

The following equality establishes the similarity between the operators $\mathcal{L}_{c, a_{i j}, \varphi_{i j}}$ and $\mathcal{L}_{c}$ :

$$
\left(\prod_{i<j} \chi_{i j}^{*}\right) \mathcal{L}_{c, a_{i j}, \varphi_{i j}}\left(\prod_{i<j} \chi_{i j}\right)=\mathcal{L}_{c} .
$$

\footnotetext{
${ }^{2}$ The case of negative $a_{i j}$ can be treated as well by changing the phase by $\pi$
} 
The latter equation implies that if the function $\psi$ is an eigenfunction of the operator $\mathcal{L}_{c}$ then the function

$$
\left(\prod_{i<j} \chi_{i j}\right) \psi
$$

is an eigenfunction for the operator $\mathcal{L}_{c, a_{i j}, \varphi_{i j}}$. It follows in particular that all such operators have the same negative spectrum. This explains why the ground state calculated by F.A.B. Coutinho et all [1] for the operator $\mathcal{L}_{c, a, \pm \pi}$ coincides with the result of C.N Yang [21]. In the case $a_{i j} \equiv 1$, the operators are unitary equivalent.

Consider the three body problem. The eigenfunctions for the operator $\mathcal{L}_{c}$ have been first calculated by J.B.McGuire [19]. There are two types of eigenfunctions: those generated by incoming plane waves and those generated by incoming surface waves concentrated near the lines $l_{i j}$. The lines $l_{i j}$ divide the plane of zero total momentum into 6 sectors (see 19 for details). The eigenfunctions of the first type corresponding to the energy $E=k_{1}^{2}+k_{2}^{2}+k_{3}^{3}$ are given in each sector by the set of plane waves

$$
\begin{aligned}
& \psi\left(x_{1}, x_{2}, x_{3}\right)= \\
& A_{123} \exp i\left(k_{1} x_{1}+k_{2} x_{2}+k_{3} x_{3}\right)+A_{132} \exp i\left(k_{1} x_{1}+k_{3} x_{2}+k_{2} x_{3}\right) \\
& +A_{213} \exp i\left(k_{2} x_{1}+k_{1} x_{2}+k_{3} x_{3}\right)+A_{231} \exp i\left(k_{2} x_{1}+k_{3} x_{2}+k_{1} x_{3}\right) \\
& +A_{312} \exp i\left(k_{3} x_{1}+k_{1} x_{2}+k_{2} x_{3}\right)+A_{321} \exp i\left(k_{3} x_{1}+k_{2} x_{2}+k_{1} x_{3}\right)
\end{aligned}
$$

with the amplitudes depending on the sector. These amplitudes can be determined by the boundary conditions. Similar formula holds for the $N$-body operators, The function $\left(\prod_{i<j} \chi_{i j}\right) \psi$ is of the same type but satisfies boundary conditions (13). It is straightforward to show that the latter function is an eigenfunction of the operator $\mathcal{L}_{c, a_{i j}, \varphi_{i j}}$. Therefore in addition to the few body system in one dimension with equal two body delta interactions there exists a $2\left(\begin{array}{l}N \\ 2\end{array}\right)+1$-parameter family of few-body problems with point interactions, that can be solved by McGuire's method. Let us consider the self-adjoint problem, i.e. when all $a_{i j}=1$. Then the family of $N$-body self-adjoint operators possessing McGuire solution is described by $\left(\begin{array}{l}N \\ 2\end{array}\right)+1$ real parameters. This is in contradiction to [11, where it has been stated that no diffraction occurs for the scattering states for the three-body Hamiltonian with point two-body interactions only in the cases $e^{i \varphi}= \pm 1$. The same condition was obtained by investigating the $N$ body problem using the Yang-Baxter equation 11. The Yang-Baxter equation is derived for particles having boson or fermion symmetry. It is obvious that nontrivial phases $\varphi \neq 0, \pi$ cannot occur for such particles.

Note that each operator $\mathcal{L}_{c, a_{i j}, \varphi_{i j}}$ is similar to $\mathcal{L}_{c}$. The operators are unitary equivalent if $a_{i j}=1$. We think that the total many parameter family of operators can play an important role for the investigation of $N$-particle Hamiltonians with a time dependent interaction between the particles. 


\section{References}

[1] S. Albeverio, L. Dąbrowski, and S-M. Fei, Int. J. Mod. Phys. B 14, 721 (2000).

[2] S. Albeverio, L. Dąbrowski, and P. Kurasov, Lett. Math. Phys. 45, 33 (1998).

[3] S. Albeverio, F. Gesztesy, R. Høegh-Krohn, H. Holden, Solvable models in quantum mechanics, Springer, 1988.

[4] F. Gesztesy and W. Kirsch, J. Reine Angew. Math. 362, 28-50 (1985).

[5] S. Albeverio and P. Kurasov, J. Func. Anal. 148, 152-169 (1997).

[6] S. Albeverio and P. Kurasov, Singular perturbations of differential operators, Cambridge Univ. Press, 2000 (London Mathematical Society Lecture Notes 271).

[7] F.A. Berezin and L.D. Faddeev, Soviet Math. Dokl. 2, 372 (1961) ( Math. USSR Dokl. 137, 1011 (1961)).

[8] V.S. Buslaev, S.P. Merkuriev, and S.P. Salikov, Problemu Mat. Fiz., vup. 9, Leningrad Univ. Press, 1979 (In Russian).

[9] P.R. Chernoff, R.J. Hughes, J. Funct. Anal. 111, 97 (1993).

[10] F.A.B. Coutinho, Y. Nogami, and L. Tomio, J. Phys. A 32, L133 (1999).

[11] F.A.B. Coutinho, Y.Nogami, and L. Tomio, J. Phys. A 32, 4931 (1999).

[12] M. Gaudin, La fonction d'onde de Bethe, Masson, 1983.

[13] D.J. Griffiths, J. Phys. A: Math. Gen. 26, 2265-2267 (1993).

[14] P. Kurasov, J. Math. Anal. Appl. 201, 297 (1996).

[15] P. Kurasov, Rev. Math. Phys. 9, 853 (1997).

[16] P. Kurasov, J. Boman, Proc. AMS 126, 1673 (1998).

[17] E. Lieb and W.Liniger, Phys. Rev. (2) 130, 1605 (1963).

[18] E. Lieb and W.Liniger, Phys. Rev. (2) 134, A312 (1964).

[19] J.B. McGuire, J. Math. Phys. 5, 622 (1964).

[20] P. Šeba, Czechoslovak J. Phys. B 36, 667 (1986).

[21] C.N. Yang, Phys. Rev. 168, 1920 (1968). 